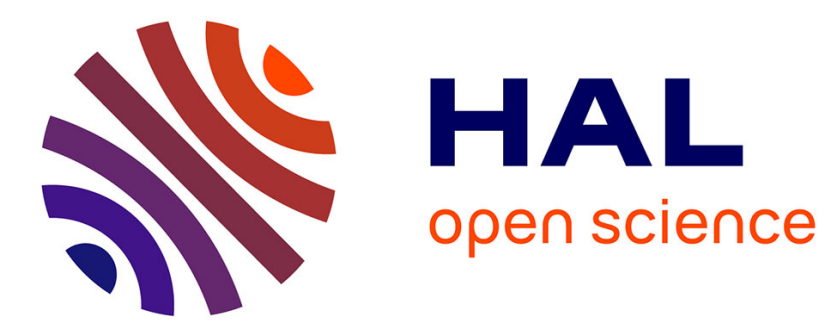

\title{
Ultrafast control of anisotropy
}

Eric Collet

\section{- To cite this version:}

Eric Collet. Ultrafast control of anisotropy: SINGLE-MOLECULE MAGNETS. Nature Chemistry, 2020, 12 (5), pp.429-430. 10.1038/s41557-020-0462-z . hal-02562752

\section{HAL Id: hal-02562752 \\ https://hal.science/hal-02562752}

Submitted on 11 May 2020

HAL is a multi-disciplinary open access archive for the deposit and dissemination of scientific research documents, whether they are published or not. The documents may come from teaching and research institutions in France or abroad, or from public or private research centers.
L'archive ouverte pluridisciplinaire HAL, est destinée au dépôt et à la diffusion de documents scientifiques de niveau recherche, publiés ou non, émanant des établissements d'enseignement et de recherche français ou étrangers, des laboratoires publics ou privés. 


\section{Ultrafast control of anisotropy}

Single-molecule magnets are able to store information through their magnetic anisotropy, making them very promising systems for memory applications. Now, femtosecond-laser-initiated molecular dynamics that modulate magnetic anisotropy have been observed, paving the way for operation on ultra-fast timescales.

\section{Eric Collet}

Univ Rennes, CNRS, IPR (Institut de Physique de Rennes) - UMR 6251, F-35000 Rennes, France

e-mail: eric.collet@univ-rennes1.fr

Twitter: @rx_collet

Single-molecule magnets (SMM) represent a molecular approach to nanomagnets. These metal-organic compounds exhibit - below a certain blocking temperature - magnetic hysteresis at the molecular scale and retain magnetisation in the absence of a magnetic field. SMMs have a bistable magnetic ground state and the associated energy barrier arises from a combination of a high-spin ground state and significant axial anisotropy of that ground state, making them promising candidates for the next generation of information storage devices. Since the first report on a single-molecule magnet, ${ }^{1}$ efforts in this field have primarily focused on raising the operating temperatures in order to enable applications in magnetic memory. ${ }^{2}$ Among strategies for increasing the scope of applications for SMM, several groups have explored various ways to control their magnetization via an external stimulus, such as pressure, electric potential, light or the coordinating solvent. ${ }^{3}$ Now, writing in Nature Chemistry, Johansson and colleagues have explored the ultrafast dynamics of magnetic control when initiated using femtosecond light excitation.

Light is a particularly intriguing tool, which could potentially enable remote and rapid control of magnetic properties. For example, studies of a Prussian blue analogue showed that, in the photoinduced chargetransfer state, its magnetic relaxation slows down, which is characteristic of superparamagnetism behaviour. ${ }^{4}$ Also, light has been used to switch SMM behaviour on and off. The behaviour was observed in an iron(II) spin-crossover system, converting between low spin ( $S=0$, off) to high spin $(S=2$, on) configurations under visible light irradiation. Its anisotropy provides SMM behaviour in the photoinduced HS state, thus giving rise to tristability $(S=0$ and \pm 2 ) and opening the way for using SMM for ternary information storage. ${ }^{5}$ Another approach connected a bispyridyl dithienylethene (DTE) unit with a highly anisotropic dysprosium-based single-molecule magnet [Dy(Tppy)F-(pyridine) $\left.{ }_{2}\right] \mathrm{PF}_{6}$. The photoisomerization of the bridging DTE ligand modulates the relaxation. ${ }^{6}$

Johansson and colleagues now bring a distinct perspective by taking the study of SMM optical excitation to the ultrafast domain. They use pulsed laser excitation to modulate the molecular anisotropy on the femtosecond timescale ( $\left.1 \mathrm{fs}=10^{-15} \mathrm{~s}\right)-$ the molecular anisotropy is what gives the magnetisation a preferred direction - and use femtosecond pump-probe spectroscopy to study the resulting ultrafast electronic and structural dynamics. In this technique, a femtosecond laser 'pump' beam is used to excite the sample, generating a non-equilibrium state, and another 'probe' beam is used to monitor the pumpinduced changes in absorption as a function of the time between the pulses (Figure 1). The absorption is modulated by both electronic and structural reorganizations within the molecule, which provides key information about the dynamic processes occurring and the adjustable pump and probe wavelengths allow for selectively probing the electronic or structural dynamics. This powerful technique is intensively used in coordination chemistry. For example it enabled the observation of vibrational coherence in the excited-state dynamics of $\mathrm{Cr}(\mathrm{acac})_{3}$ during sub-200 fs intersystem crossing ${ }^{7}$ and the light-induced excited spin-state trapping dynamics of $\mathrm{Fe}$ (II) complexes ${ }^{8}$, which is driven by the activation and damping of the vibrational wavepacket along the $\mathrm{Fe}-$ ligand reaction coordinate.

Johansson and co-workers have now studied the response to femtosecond-laser excitation of the SMM $\left[\mathrm{Mn}(\mathrm{III})_{3} \mathrm{O}(\mathrm{Et}-\mathrm{sao})_{3}(\mathrm{\beta}-\mathrm{pic})_{3}\left(\mathrm{ClO}_{4}\right)\right]\left(\mathrm{Mn}_{3} ; \mathrm{Fig} .1\right)$, as well as mononuclear $(\mathrm{Mn})$ and hexa-nuclear $\left(\mathrm{Mn}_{6}\right)$ derivatives. The optical pump excitation process promotes one electron from the $d_{z^{2}}$ to the $d_{x^{2}-y^{2}}$ orbital and the subsequent dynamics were monitored in real time. They observed oscillatory patterns superimposed on the timedependent absorption change, which is associated with coherent structural dynamics. This is in contrast to incoherent molecular vibrations that are populated at thermal equilibrium and do not have a phase relationship - such dynamics result in a constant absorption statistically averaged over the huge ensemble of probed molecules. Femtosecond excitation, however, can launch simultaneously the molecules on a deterministic pathway along a reaction coordinate on the potential energy surface. The associated molecular oscillations along the mode in the photoinduced potential, which modulate absorption, are then in phase because the laser pulse is shorter than the oscillating period, resulting in the structural dynamics of the molecules behaving coherently.

Based on complementary measurements and calculations, the co-workers attribute the oscillations to a reaction coordinate mode, stabilizing the $d_{x^{2}-y^{2}}$ state and shifting consequently the distortion created by the Jahn-Teller (JT) effect from axial to equatorial (Figure 1). This transformation towards a switched JT state is associated with a collective in-phase stretching mode along the JT axis (Figure 1; top right). The triangular structure of $\mathrm{Mn}_{3}$, where the bridged $\mathrm{Mn}$ have similar anisotropy axis, enhances the coherence between the $\mathrm{Mn}$ sites. Both the ultrafast and displacive nature of the structural response to optical electronic excitation are responsible for the observed coherent dynamics. Similar observations are reported for the $\mathrm{Mn}$ and $\mathrm{Mn}_{6}$ samples and the results of Johansson and colleagues demonstrate that the magnetic anisotropy - and consequently the 
SMM behaviour - can be coherently modulated on the 100 fs timescale.

The approach used by Johansson and colleagues using an ultrashort light pulse in the visible range for ultrafast tuning of SMM anisotropy - does not allow the control or reversal of spin, because the light pulse used does not couple to magnetic order. Thus, new laser-based techniques generating terahertz $\left(1 \mathrm{THz}=10^{12} \mathrm{~Hz}\right)$ electromagnetic fields may be considered in the future to rapidly reverse a SMM's polarity. Indeed, it was recently shown in the antiferromagnetic $\mathrm{TmFeO}_{3}$ (thulium orthoferrite) that $\mathrm{THz}$ pulses allow coherent steering of spins through their coupling with the $\mathrm{THz}$ electric field, ${ }^{9}$ as high as $1 \mathrm{MV} \mathrm{cm}^{-1}$. For the moment, this technique is mainly used for hard-condensed matter. THz excitation of SMM is for the moment a 'Tera' incognita, but THz pulses may provide strong enough interaction to steer the magnetization of all the spins over a potential energy barrier and into a new orientation in just picoseconds. This may be the ultimate way to write and store information at both ultra-small spatial and ultrafast temporal molecular scales. But for the time being, the insights from Johansson and colleagues certainly set us on the right track and shed new light on the ultrafast dynamics of such singlemolecule magnets.

Figure 1: Optical pump-probe study of $\mathrm{Mn}_{3}$ single-molecule magnets. After fs optical excitation (green pulse) promoting one electron from $d_{z^{2}}$ to $d_{x^{2}-y^{2}}$ orbitals, the evolution of the system is tracked by time-resolved absorption change (red curve). The data reveal a coherent oscillation due to the structural reorganization from axial JahnTeller deformation (grey arrow when the $d_{z^{2}}$ state is occupied) to equatorial (grey disc when the $d_{x^{2}-y^{2}}$ state is occupied), around the constituting $\mathrm{Mn}$ (III) ions. The coherent dynamics corresponds to a collective stretching of the bond lengths along the JT axis for all three Mn ions as illustrated (top right).

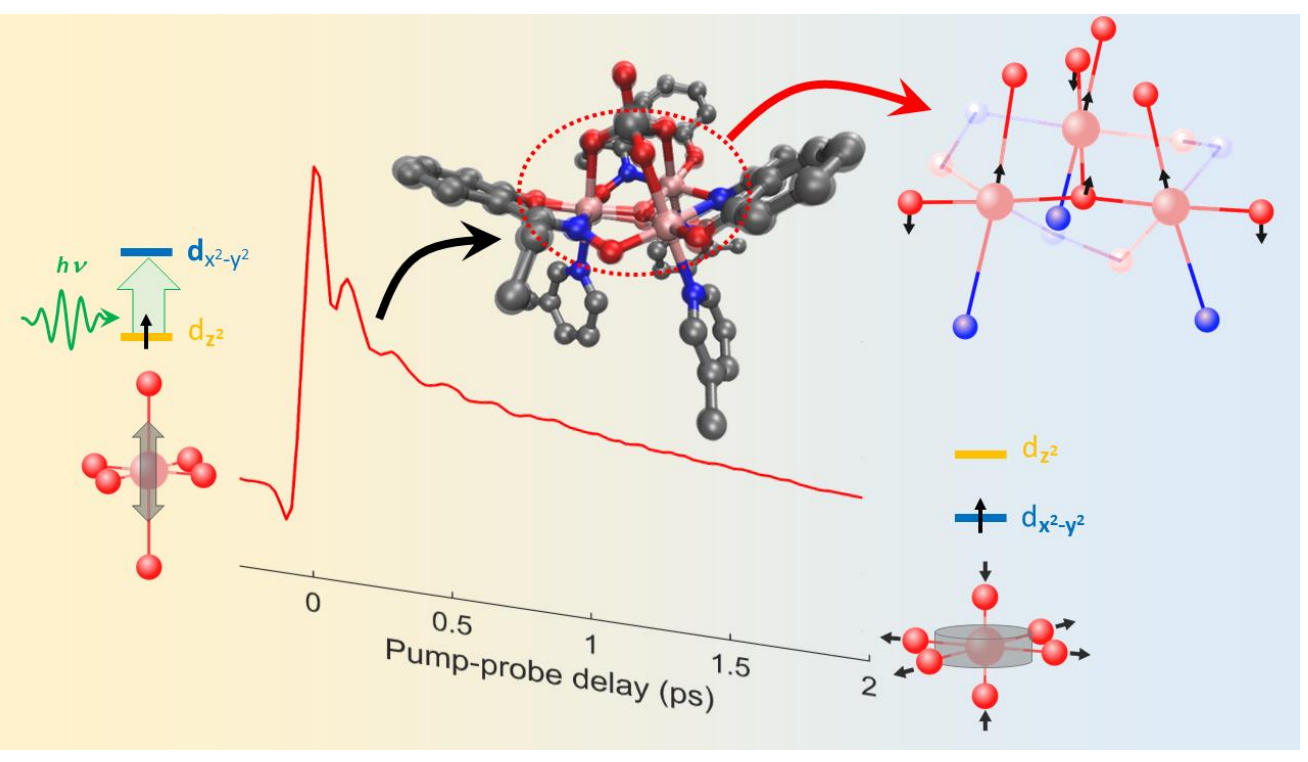

Caneschi, A. et al. Alternating current susceptibility, high field magnetization, and millimeter band EPR evidence for a ground $\mathrm{S}=10$ state in [Mn12O12(Ch3COO)16(H2O)4].2CH3COOH.4H2O. Journal of the American Chemical Society 113, 5873-5874, doi:10.1021/ja00015a057 (1991).

2 Guo, F.-S. et al. Magnetic hysteresis up to 80 kelvin in a dysprosium metallocene single-molecule magnet. Science 362, 1400-1403, doi:10.1126/science.aav0652 (2018).

3 Jiang, W. et al. Switching single chain magnet behavior via photoinduced bidirectional metal-to-metal charge transfer. Chem Sci 9, 617-622, doi:10.1039/C7SC03401F (2018). Nihei, M. et al. A Light-Induced Phase Exhibiting Slow Magnetic Relaxation in a Cyanide-Bridged [Fe4Co2] Complex. Angewandte Chemie International Edition 51, 6361-6364, doi:10.1002/anie.201202225 (2012). Feng, X. et al. Tristability in a Light-Actuated Single-Molecule Magnet. Journal of the American Chemical Society 135, 15880-15884, doi:10.1021/ja407332y (2013).

6 Hojorat, M. et al. Hysteresis Photomodulation via Single-Crystal-to-Single-Crystal Isomerization of a Photochromic Chain of Dysprosium Single-Molecule Magnets. Journal of the American Chemical Society 142, 931-936, doi:10.1021/jacs.9b10584 (2020).

7 Schrauben, J. N., Dillman, K. L., Beck, W. F. \& McCusker, J. K. Vibrational coherence in the excited state dynamics of $\mathrm{Cr}(\mathrm{acac})(3)$ : probing the reaction coordinate for ultrafast intersystem crossing. Chem Sci 1, 405410, doi:Doi 10.1039/COsc00262c (2010). Lemke, H. T. et al. Coherent structural trapping through wave packet dispersion during photoinduced spin state switching. Nat. Commun. 8, 15342 (2017).

9 Schlauderer, S. et al. Temporal and spectral fingerprints of ultrafast all-coherent spin switching. Nature 569, 383-387, doi:10.1038/s41586-019-1174-7 (2019). 